\title{
Combining Eliashberg Theory with Density Functional Theory for the Accurate Prediction of Superconducting Transition Temperatures and Gap Functions
}

\author{
A. Sanna® \\ Max-Planck-Institut für Mikrostrukturphysik, Weinberg 2, D-06120 Halle, Germany \\ C. Pellegrini@ and E. K. U. Gross \\ Fritz Haber Center for Molecular Dynamics, Institute of Chemistry, The Hebrew University of Jerusalem, \\ Jerusalem 91904, Israel
}

(Received 3 January 2020; revised 10 May 2020; accepted 18 June 2020; published 27 July 2020)

\begin{abstract}
We propose a practical alternative to Eliashberg equations for the $a b$ initio calculation of superconducting transition temperatures and gap functions. Within the recent density functional theory for superconductors, we develop an exchange-correlation functional that retains the accuracy of Migdal's approximation to the many-body electron-phonon self-energy, while having a simple analytic form. Our functional is based on a parametrization of the Eliashberg self-energy for a superconductor with a single Einstein frequency, and enables density functional calculations of experimental excitation gaps. By merging electronic structure methods and Eliashberg theory, the present approach sets a new standard in quality and computational feasibility for the prediction of superconducting properties.
\end{abstract}

DOI: 10.1103/PhysRevLett.125.057001

One of the great challenges of modern condensed-matter theory is the prediction of material-specific properties of superconductors, such as the critical temperature $T_{C}$ and the gap at zero temperature $\Delta$. The model of Bardeen, Cooper, and Schrieffer (BCS) accounts for the universal behavior of all conventional superconductors, but is not able to describe material dependent properties [1]. A major step toward a first-principles approach to superconductivity is Eliashberg theory [2]. Within the latter, all the relevant quantities that govern the superconducting properties of a material are calculated in the Nambu Green's function formalism [3,4] using the analog of $G W$ as approximation for the self-energy, where $W$ refers to the screened Coulomb interaction, plus a renormalized phonon propagator. By virtue of Migdal's theorem [2,4,5], which states that the neglected terms in the perturbation expansion of the self-energy are of the order of the square root of the electron to ion mass ratio, this method describes electron-phonon coupling effects very accurately. However, the treatment of Coulomb correlations is much harder, and, in practice, the electron-electron repulsion is usually modeled by an adjustable parameter $\mu *$, chosen as to reproduce the experimental $T_{C}[6,7]$. In its common applications, thus,

Published by the American Physical Society under the terms of the Creative Commons Attribution 4.0 International license. Further distribution of this work must maintain attribution to the author(s) and the published article's title, journal citation, and DOI. Open access publication funded by the Max Planck Society.
Eliashberg theory cannot be considered a truly $a b$ initio theory either.

Combining good accuracy with moderate numerical effort, density functional theory (DFT) is the method of choice for $a b$ initio calculations of material-specific properties in the normal state. Following the discovery of hightemperature superconductors, the formal framework of DFT has been generalized to describe the superconducting phase of matter [8]. Compared to traditional many-body approaches, DFT for superconductors (SCDFT) yields two major advantages: the computational feasibility of the equations and the absence of any empirical parameter, such as the $\mu *$ of Eliashberg theory. In SCDFT, both the normal density $n(\mathbf{r})$ and the superconducting order parameter $\chi\left(\mathbf{r}, \mathbf{r}^{\prime}\right)$ of the system are reproduced by a noninteracting Kohn-Sham (KS) system, where the exchangecorrelation (xc) effects on superconductivity are included via the pairing potential $\Delta_{s}\left(\mathbf{r}, \mathbf{r}^{\prime}\right)[n, \chi]$. Within the so-called decoupling approximation [9-11], the diagonal matrix elements of $\Delta_{s}$ with respect to the normal-state KS Bloch orbitals, $\Delta_{s k}=\left\langle\varphi_{k}\left|\Delta_{s}\right| \varphi_{k}\right\rangle$, play the role of a BCS gap function in the quasiparticle excitation spectrum, $E_{k}=\sqrt{\xi_{k}^{2}+\left|\Delta_{s k}\right|^{2}}$, where $\xi_{k}$ are the eigenvalues of the normal-state KS orbitals with index $k=(\mathbf{k}, n)$, i.e., Bloch vector $\mathbf{k}$ and band index $n$. The KS system is fully determined by solving the BCS-like gap equation,

$$
\Delta_{s k}=-\mathcal{Z}_{k} \Delta_{s k}-\frac{1}{2} \sum_{k^{\prime}} \mathcal{K}_{k, k^{\prime}} \frac{\tanh \left(\frac{\beta}{2} E_{k^{\prime}}\right)}{E_{k^{\prime}}} \Delta_{s k^{\prime}},
$$


where $\beta$ is the inverse temperature. The kernel of this equation consists of two parts: a diagonal part $\mathcal{Z}_{k}$, which acts as the renormalization term in the Eliashberg equations, and a nondiagonal part, $\mathcal{K}_{k, k^{\prime}}=\mathcal{K}_{k, k^{\prime}}^{e e}+\mathcal{K}_{k, k^{\prime}}^{\mathrm{ph}}$, which can be interpreted as the interaction responsible for the formation of Cooper pairs.

As for any DFT scheme, the success of the theory depends primarily on the availability of reliable exchangecorrelation functionals. However, contrary to ordinary DFT, where a variety of functionals has appeared over the years, only one approximation has been proposed for the superconducting state $[10,11]$. By means of the optimized effective potential method, the exchange-correlation kernels of Eq. (1) have been evaluated from the lowest order exchange diagram of the electron self-energy stemming from the Coulomb and phonon-mediated interactions. The functional thereby obtained, here referred to as LM2005, has opened up to fully $a b$ initio calculations of $T_{C}$ with relevant results [12-18]. In particular, thanks to the low computational cost of the method, it has enabled the study of the full k-dependent anisotropy in the superconducting gap [19-25].

However, the LM2005 functional suffers from a major drawback: by construction it neglects higher order processes that in Eliashberg theory are included by the selfconsistent dressing of the electron Green's function in the self-energy diagram. In other words, the functional approximation is not validated by Migdal's theorem, which makes it of questionable accuracy for treating electronphonon coupling effects. Attempts to go beyond the exchange-only approximation have motivated a correction to the original $\mathcal{Z}$ kernel $[10,11,26]$. Nevertheless, the corrected functional yields $T_{C}$ 's that are systematically too low $[14,19,21]$ (the most striking example is $\mathrm{MgB}_{2}$, which is predicted with a $T_{C}$ of $25 \mathrm{~K}$, against the experimental value of $39 \mathrm{~K}$ [24]). In this Letter, we overcome this problem by constructing an exchangecorrelation self-energy that retains the accuracy of the many-body Eliashberg self-energy, while having a simple analytic form.

We adopt the $2 \times 2$ Nambu representation for the Matsubara Green's function in momentum space,

$$
\bar{G}\left(k, \omega_{n}\right)=\left(\begin{array}{cc}
G\left(k, \omega_{n}\right) & -F\left(k, \omega_{n}\right) \\
-F^{*}\left(k, \omega_{n}\right) & -G\left(-k,-\omega_{n}\right)
\end{array}\right),
$$

where $G\left(k, \omega_{n}\right)$ and $F\left(k, \omega_{n}\right)$ are, respectively, the normal and anomalous components. The expressions for the propagators of the unperturbed $\mathrm{KS}$ system read as $G^{s}\left(k, \omega_{n}\right)=-\left(i \omega_{n}+\xi_{k}\right) /\left(\omega_{n}^{2}+E_{k}^{2}\right) \quad$ and $\quad F^{s}\left(k, \omega_{n}\right)=$ $-\Delta_{s k} /\left(\omega_{n}^{2}+E_{k}^{2}\right)$. Moreover, the Matsubara phonon propagator has the standard form $D_{q, \eta}\left(\nu_{n}\right)=-2 \omega_{q, \eta} /$ $\left(\nu_{n}^{2}+\omega_{q, \eta}^{2}\right)$, where $q\left(\equiv \mathbf{k}-\mathbf{k}^{\prime}\right)$ is the phonon wave vector and $\eta$ is the mode index.
Using Dyson's equation, and the fact that the superconducting order parameter is given by

$$
\chi(k)=-\frac{1}{\beta} \sum_{n} F\left(k,-\omega_{n}\right)=-\frac{1}{\beta} \sum_{n} F^{s}\left(k,-\omega_{n}\right),
$$

for both the interacting system and the KS system, we obtain the Sham-Schlüter equation [26,27],

$$
\frac{1}{\beta} \sum_{n}\left[\bar{G}^{s}\left(k,-\omega_{n}\right) \tilde{\Sigma}_{\mathrm{xc}}\left(k,-\omega_{n}\right) \bar{G}\left(k,-\omega_{n}\right)\right]_{12}=0,
$$

where $\tilde{\Sigma}_{\mathrm{xc}}\left(k, \omega_{n}\right)=\bar{\Sigma}_{\mathrm{xc}}\left(k, \omega_{n}\right)-\left(\begin{array}{cc}0 & \Delta_{s k} \\ \Delta_{s k}^{*} & 0\end{array}\right)$. Here, the irreducible Nambu self-energy $\bar{\Sigma}_{\text {xc }}$ can be decomposed into a (purely anomalous) Coulomb part, and a part involving the phonon-induced interaction. The Coulomb self-energy part accounts for the reduction in the superconducting gap due to the electronically screened Coulomb interaction. We assume that this effect is described by the anomalous Hartree potential in the static random-phase approximation (RPA) [19,28], and focus on developing approximations for the phononic contribution to $\Delta_{s}$.

It is known from Migdal's theorem that, to a good accuracy, electron-phonon vertex corrections can be neglected in the perturbative expansion of the electron self-energy. However, self-energy insertions of phononic origin should be included to have a consistent description of phonon-mediated effects. Accordingly, we approximate the phononic part of $\bar{\Sigma}_{\mathrm{xc}}$ with the Eliashberg self-energy,

$$
\begin{aligned}
\bar{\Sigma}_{\mathrm{xc}}\left(k, \omega_{n}\right)= & -\frac{1}{\beta} \sum_{k^{\prime}, n^{\prime}, \eta} \sigma_{3} \bar{G}\left(k^{\prime}, \omega_{n^{\prime}}\right) \sigma_{3} \\
& \times\left|g_{k, k^{\prime}, \eta}\right|^{2} D_{q, \eta}\left(\omega_{n}-\omega_{n^{\prime}}\right),
\end{aligned}
$$

where $\sigma_{3}$ is the Pauli matrix $\left(\begin{array}{cc}1 & 0 \\ 0 & -1\end{array}\right)$, and $g_{k, k^{\prime}, \eta}$ are the electron-phonon matrix elements [29]. After inserting the expression above for the self-energy, Eq. (3) reduces to the compact form,

$$
\Delta_{s k}=\frac{\tau_{k}^{K}-\tau_{k}^{L}}{\tau_{k}^{I}},
$$

which involves the following Matsubara frequency summations:

$$
\begin{aligned}
& \tau_{k}^{K}=\frac{1}{\beta} \sum_{n}[ F^{s}\left(k, \omega_{n}\right) \Sigma_{\mathrm{xc}}^{12}\left(k, \omega_{n}\right) F\left(k, \omega_{n}\right) \\
&\left.-G^{s}\left(k, \omega_{n}\right) \Sigma_{\mathrm{xc}}^{12}\left(k, \omega_{n}\right) G\left(k,-\omega_{n}\right)\right], \\
& \tau_{k}^{L}=\frac{1}{\beta} \sum_{n}\left[G^{s}\left(k, \omega_{n}\right) \Sigma_{\mathrm{xc}}^{11}\left(k, \omega_{n}\right) F\left(k, \omega_{n}\right)\right. \\
&\left.+F^{s}\left(k, \omega_{n}\right) \Sigma_{\mathrm{xc}}^{11}\left(k,-\omega_{n}\right) G\left(k,-\omega_{n}\right)\right],
\end{aligned}
$$


$\tau_{k}^{I}=\frac{1}{\beta} \sum_{n}\left[F^{s}\left(k, \omega_{n}\right) F\left(k, \omega_{n}\right)-G^{s}\left(k, \omega_{n}\right) G\left(k,-\omega_{n}\right)\right]$.

Here, $\Sigma_{\mathrm{xc}}^{11}$ and $\Sigma_{\mathrm{xc}}^{12}$ are, respectively, the normal and anomalous components of the Eliashberg self-energy Eq. (4).

Equation (5) is formally correct, but not useful in practice since solving it requires first calculating the interacting Nambu Green's function. Thus, one must rely on an approximation scheme. The straightforward approach would be replacing $\bar{G}_{k, n}$ by $\bar{G}_{k, n}^{s}$ on all the occurrences to obtain a closed KS equation for $\Delta_{s k}$. This approach yields the LM2005 functional. On the other hand, setting $\bar{\Sigma}_{\mathrm{xc}}\left[\bar{G}_{k, n}\right]=\bar{\Sigma}_{\mathrm{xc}}\left[\bar{G}_{k, n}^{s}\right]$ is not justified by Migdal's theorem, resulting in an uncontrolled approximation for $\Delta_{s k}$.

We now introduce a simple model for which Eq. (5) can be evaluated exactly, i.e., a conventional superconductor with a simplified phonon system described by a single Einstein mode of frequency $\omega_{\mathrm{ph}}$. We assume that the superconductor is isotropic, so that the pairing potential does not depend on the direction of $\mathbf{k}$, but only on the energy, $\Delta_{s k}=\Delta_{s}\left(\xi_{k}\right)$. The electron-phonon matrix elements in Eq. (4) then reduce to $g_{k, k^{\prime}, \eta}=\sqrt{\lambda \omega_{\mathrm{ph}} /\left(2 N_{F}\right)}$, where $\lambda$ is the (isotropic) coupling function and $N_{F}$ is the density of states at the Fermi energy. Here, we choose $\lambda=1$ and $\omega_{\mathrm{ph}}=60 \mathrm{meV}$. The numerically exact $\Delta_{s}(\xi)$ for the model is shown in Fig. 1 for several temperature ratios $T / T_{C}$. As one can see, the overall shape of $\Delta_{S}$ as a function of $\xi$ is independent of the temperature: the potential has a vanishing first derivative at the Fermi energy, in correspondence of a local minimum, and rapidly approaches zero at a few times the Debye energy from the Fermi surface.

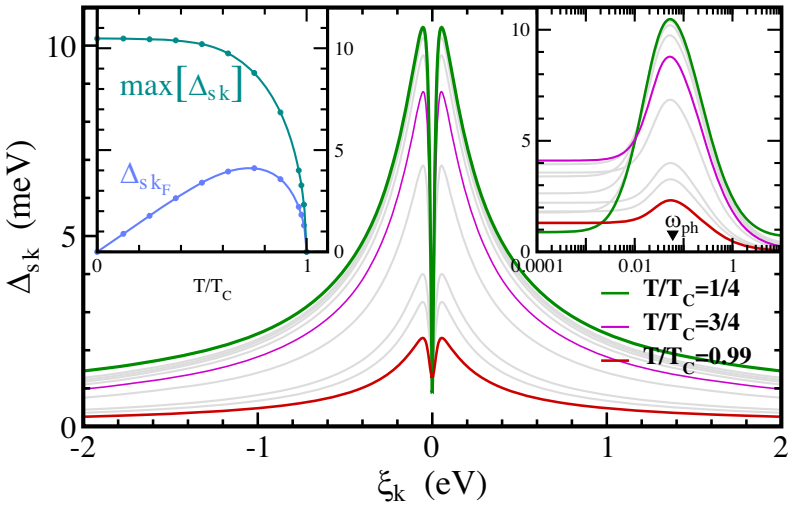

FIG. 1. Main plot and semilog scale inset at top right: Energy and temperature dependence of the phonon-only KS pair potential $\Delta_{s k}$ in the Eliashberg approximation for an electronic system interacting with an Einstein phonon mode of frequency $\omega_{\mathrm{ph}}=$ $60 \mathrm{meV}$ and coupling strength $\lambda=1$. Inset at top left: Temperature dependence of the peak and Fermi value of the KS gap.
The exact result that we want to emphasize here is the vanishing of $\Delta_{s}$ at the Fermi level for values of $T$ approaching zero. This behavior is in striking contrast with that shown by the LM2005 functional [10]. The latter, in fact, relies on the assumption that the exact $\Delta_{s}$ has to reproduce the experimental excitation gap (which is indeed maximum at $T=0$, and decreases monotonically with increasing temperature, reaching the value of zero at $T=T_{C}$ ). However, the nonphysical behavior of $\Delta_{s}$ should not be a surprise. The main point is that, like in normal-state DFT, the KS system is not constructed to give the excitation spectrum of the real interacting system, but only its density. In the following, we thus set aside the interpretation of $\Delta_{s}$ as a true gap function, and work out an approximation based on the exact result for our reference system.

We substitute the interacting Green's functions in Eq. (5) by their KS counterparts selectively, and use our model to benchmark the resulting approximations. We find that, although this substitution is generally allowed, it has a major impact on the prediction of $T_{C}$ when applied to the off-diagonal components of $\bar{\Sigma}_{\text {xc }}$ (i.e., the anomalous irreducible self-energies), yielding unphysical results. To cure this flaw of the approximation, we introduce an auxiliary anomalous self-energy in the following form:

$$
\begin{aligned}
\Sigma_{k, n}^{12}\left[\bar{G}^{s}\right]= & -\frac{1}{\beta} \sum_{k^{\prime}, n^{\prime}, \eta}\left(\frac{\gamma_{1} \Delta_{s k^{\prime}}}{\omega_{n^{\prime}}^{2}+\xi_{k^{\prime}}^{2}+\gamma_{3} \Delta_{s k^{\prime}}^{2}} \frac{\gamma_{2}^{2} \omega_{q, \eta}^{2}}{\omega_{n^{\prime}}^{2}+\gamma_{2}^{2} \omega_{q, \eta}^{2}}\right) \\
& \times\left|g_{k, k^{\prime}, \eta}\right|^{2} D_{q, \eta}\left(\omega_{n}-\omega_{n^{\prime}}\right),
\end{aligned}
$$

where $\gamma_{1}, \gamma_{2}$, and $\gamma_{3}$ are adjustable parameters. While being mathematically simple, Eq. (9) has the advantage of optimally reproducing the off-diagonal component of the Eliashberg self-energy for the model, upon a suitable choice of the parameters $\gamma_{i}$. For the Einstein spectrum, the bracketed term plays the role of $F\left(k^{\prime}, \omega_{n^{\prime}}\right)$, where the frequency dependence of the Eliashberg gap function is given by the factor $\gamma_{2}^{2} \omega_{\mathrm{ph}}^{2} /\left(\omega_{n^{\prime}}^{2}+\gamma_{2}^{2} \omega_{\mathrm{ph}}^{2}\right)$, which becomes exact in the weak coupling limit (see the Supplemental Material [30]) [34]. Using Eq. (9) for $\Sigma_{\mathrm{xc}}^{12}$ in Eq. (5), and replacing $\bar{G}$ by $\bar{G}^{s}$ elsewhere, we are left with a closed selfconsistent equation for $\Delta_{s k}$. Notably, the latter involves only Matsubara sums that can be evaluated analytically, which avoids the computational complexity of solving the Eliashberg gap equation.

In proximity of $T_{C}$, the obtained $\mathrm{KS}$ gap equation can be partially linearized in $\chi$. By recasting the simplified equation in the form (1), the phononic exchange-correlation kernels read:

$$
\begin{aligned}
\mathcal{Z}_{k} & =-\frac{1}{\tanh \left[(\beta / 2) \xi_{k}\right]} \sum_{k^{\prime}, \eta}\left|g_{k, k^{\prime}, \eta}\right|^{2} \\
& \times \frac{d}{d \xi_{k}}\left[I\left(\xi_{k}, \xi_{k^{\prime}}, \omega_{q, \eta}\right)+I\left(\xi_{k},-\xi_{k^{\prime}}, \omega_{q, \eta}\right)\right],
\end{aligned}
$$




$$
\begin{aligned}
\mathcal{K}_{k, k^{\prime}}^{\mathrm{ph}}= & \frac{\gamma_{1} \gamma_{2}}{\tanh \left[(\beta / 2) \xi_{k}\right] \tanh \left[(\beta / 2) E_{k^{\prime}}\right]} \sum_{\eta} \omega_{q, \eta}\left|g_{k, k^{\prime}, \eta}\right|^{2} \\
& \times \sum_{s_{i}= \pm} s_{1} s_{2} s_{3} J\left(\xi_{k}, s_{1} \sqrt{\xi_{k^{\prime}}^{2}+\gamma_{3} \Delta_{s k^{\prime}}^{2}}, s_{2} \omega_{q, \eta}, s_{3} \gamma_{2} \omega_{q, \eta}\right) .
\end{aligned}
$$

Here, we have defined the functions

$$
\begin{aligned}
I\left(\xi, \xi^{\prime}, \omega\right)= & f(\xi) f\left(\xi^{\prime}\right) n(\omega) \\
& \times\left[\frac{e^{\beta \xi}-e^{\beta\left(\xi^{\prime}+\omega\right)}}{\xi-\xi^{\prime}-\omega}-\frac{e^{\beta \xi^{\prime}}-e^{\beta(\xi+\omega)}}{\xi-\xi^{\prime}+\omega}\right],
\end{aligned}
$$

where $f(\xi)$ and $n(\omega)$ are, respectively, the Fermi and Bose distributions. In addition, the Coulomb part of the kernel $\mathcal{K}^{e e}$ is simply given by the matrix elements of the static RPA screened potential. It should be noted that in deriving Eq. (10) we have used the symmetric approximation proposed in Ref. [10]. Coming from the term $\tau_{k}^{L}$ of Eq. (7), this kernel accounts for phonon renormalization effects in the normal state within the exchange-only approximation. On the other hand, $\mathcal{K}^{\mathrm{ph}}$ follows from the term $\tau_{k}^{K}$ of Eq. (6), and, through Eq. (9), retains the relevant physics of the electron-phonon coupling in the superconducting state.

The exchange-correlation functional $\mathcal{K}^{\mathrm{ph}}$ depends on the three parameters $\gamma_{i}$. The straightforward way to fix these parameters to universal values would be fitting Eq. (9) to the exact Eliashberg self-energy for the model. However, for our purposes it is more convenient optimizing the prediction of $T_{C}$. This sets $\gamma_{1}=1.33$ and $\gamma_{2}=3.8$, whereas it leaves $\gamma_{3}$ free. We choose the latter to best reproduce the exact $\Delta_{s}$ as $\gamma_{3}=\gamma_{1}$. The $T_{C}$ for the model calculated from Eqs. (10) and (11) is shown in Fig. 2 as a function of the electron-phonon coupling strength $\lambda$, compared to the phonon-only results from Eliashberg theory and the LM2005 functional.

As already emphasized, within a DFT framework, the KS equations do not give direct access to the excitation spectrum of the system. Nevertheless, the Hohenberg-Kohn

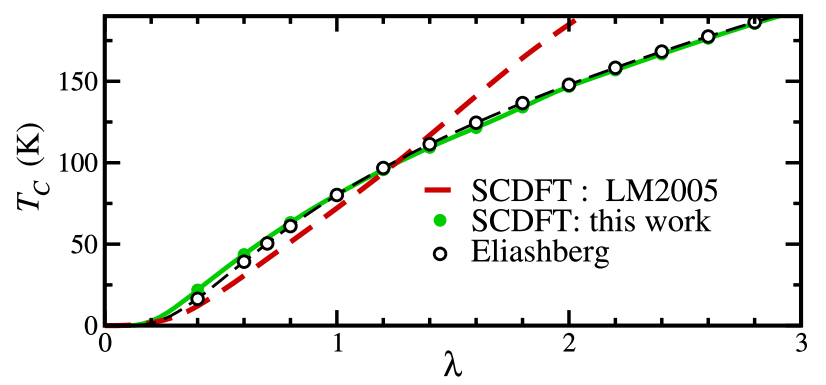

FIG. 2. Comparison of critical temperatures (Eliashberg, black; SCDFT-LM2005 [10,11], red; SCDFT-proposed functional, green) for an electronic system coupled to an Einstein phonon mode of frequency $\omega_{\mathrm{ph}}=60 \mathrm{meV}$ as a function of the electronphonon coupling $\lambda$. theorems for SCDFT [8] state that the superconducting gap is a functional of the ground-state normal and anomalous densities. In this regard, Eq. (9) provides us with a straightforward approximation to the gap function,

$$
\Delta_{k, n}=\Sigma_{k, n}^{12}\left[\bar{G}^{s}\right] Z_{k, n}^{-1}\left[\bar{G}^{s}\right],
$$

and to the experimental superconducting gap, that is the value of $\Delta_{k, n}$ at the Fermi surface [35]. Here $Z_{k, n}\left[\bar{G}^{s}\right]=$ $1+i \Sigma_{k, n}^{11}\left[\bar{G}^{s}\right] / \omega_{n}$, where the functional $\Sigma_{k, n}^{11}\left[\bar{G}^{s}\right]$ is simply obtained by replacing $G$ with $G^{s}$ in the normal component of the Eliashberg self-energy $\Sigma_{\mathrm{xc}}^{11}$. Note that in Eq. (14) we define $\Sigma_{k, n}^{12}\left[\bar{G}^{s}\right]$ by the parameter values $\gamma_{1}=1.33$, $\gamma_{2}=3.8$, and $\gamma_{3}=1.95$, which best reproduce the Eliashberg gap for our reference system [30].

In order to assess the accuracy of the proposed functional, we calculate the gaps and transition temperatures for a set of superconductors, including elemental systems from weak to strong coupling, and high- $T_{C}$ binary compounds. In Fig. 3 we show the calculated $T_{C}$ 's and superconducting

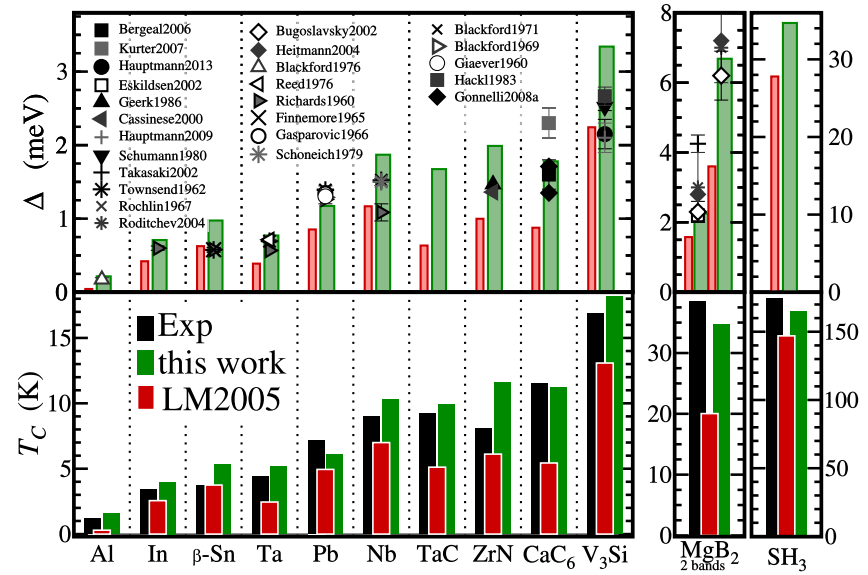

FIG. 3. Comparison between theoretical and experimental [3861] superconducting transition temperatures $\left(T_{C}\right)$ and gaps $(\Delta)$ showing that the present functional systematically improves over LM2005 [10,11]. All the materials are simulated at the calculated lattice within the PBE approximation [62]. Phonons are computed using density functional perturbation theory [29]; Coulomb scattering is calculated in static RPA. 
gaps, compared with the LM2005 and experimental results [36]. As one can see, the $T_{C}$ 's and gaps predicted by the new functional are in excellent agreement with the experimental data for all the chosen materials, improving significantly over the LM2005 approximation. It is important to note that, whereas for purely phononic pairing the LM2005 functional underestimates $T_{C}$ at weak coupling, and overestimates it at strong coupling (see Fig. 2), in realistic calculations it tends to underestimate the experimental $T_{C}$. This deficiency of the LM2005 functional can be traced back to an incorrect treatment of the $\tau_{k}^{K}$ term of Eq. (6), which spoils the energy dependence of the superconducting gap, reducing the Coulomb renormalization process $[7,28]$. For completeness, we point out that a density matrix functional theory for superconductors [37] has been recently formulated. Owing to its formal similarity to SCDFT, we expect that this novel approach will also benefit from our functional.

In conclusion, we have proposed a novel parameter-free approach, which combines SCDFT and Eliashberg theory, for the accurate prediction of superconducting properties at a low computational cost. It is known that the experimental quasiparticle gap does not correspond to the KS singleparticle gap. Hence, to obtain the quasiparticle gap one usually resorts to many-body perturbation theory and performs, e.g., $G W$ calculations. In this Letter, we solve this fundamental problem in SCDFT by using a KS representation of the Eliashberg self-energy for a simple Einstein model to express the superconducting gap as a functional of the density. In all cases tested so far, the critical temperatures and gaps predicted by our new functional are in excellent agreement with the experimental data, while the numerical effort is significantly lower than the solution of the full Eliashberg equations [63]. The approach presented in this Letter sets a new standard for the balance of accuracy and computational cost.

A. S. acknowledges useful discussions with S. Pittalis, A. Linscheid, and J. K. Dewhurst. E. K. U. G. acknowledges financial support by the European Research Council Advanced Grant FACT (No. ERC-2017-AdG-788890).

[1] J. Bardeen, L. N. Cooper, and J. R. Schrieffer, Phys. Rev. 108, 1175 (1957).

[2] G. M. Eliashberg, J. Exp. Theor. Phys. 38, 966 (1960); Sov. Phys. JETP 11, 696 (1960).

[3] P. B. Allen and B. Mitrović, Theory of Superconducting $T_{c}$, edited by F. S. Henry Ehrenreich and D. Turnbull, Solid State Physics Vol. 37 (Academic Press, New York, 1983), pp. 1-92.

[4] S. Vonsovsky, Y. Izyumov, E. Kurmaev, E. Brandt, and A. Zavarnitsyn, Superconductivity of Transition Metals: Their Alloys and Compounds, Springer Series in Solid-State Sciences Series (Springer Limited, London, 1982).

[5] A. B. MigdalJ. Exp. Theor. Phys. 34, 1438 (1958); Sov. Phys. JETP 7, 996 (1958).
[6] P. Morel and P. W. Anderson, Phys. Rev. 125, 1263 (1962).

[7] D. J. Scalapino, J. R. Schrieffer, and J. W. Wilkins, Phys. Rev. 148, 263 (1966).

[8] L. N. Oliveira, E. K. U. Gross, and W. Kohn, Phys. Rev. Lett. 60, 2430 (1988).

[9] E. K. U. Gross and S. Kurth, Int. J. Quantum Chem. 40, 289 (1991).

[10] M. Lüders, M. A. L. Marques, N. N. Lathiotakis, A. Floris, G. Profeta, L. Fast, A. Continenza, S. Massidda, and E. K. U. Gross, Phys. Rev. B 72, 024545 (2005).

[11] M. A. L. Marques, M. Lüders, N. N. Lathiotakis, G. Profeta, A. Floris, L. Fast, A. Continenza, E. K. U. Gross, and S. Massidda, Phys. Rev. B 72, 024546 (2005).

[12] G. Profeta, C. Franchini, N. N. Lathiotakis, A. Floris, A. Sanna, M. A. L. Marques, M. Lüders, S. Massidda, E. K. U. Gross, and A. Continenza, Phys. Rev. Lett. 96, 047003 (2006).

[13] P. Cudazzo, G. Profeta, A. Sanna, A. Floris, A. Continenza, S. Massidda, and E. K. U. Gross, Phys. Rev. Lett. 100, 257001 (2008).

[14] R. Akashi, K. Nakamura, R. Arita, and M. Imada, Phys. Rev. B 86, 054513 (2012).

[15] R. Akashi and R. Arita, Phys. Rev. B 88, 054510 (2013).

[16] R. Akashi, M. Kawamura, S. Tsuneyuki, Y. Nomura, and R. Arita, Phys. Rev. B 91, 224513 (2015).

[17] A. Floris, G. Profeta, N. N. Lathiotakis, M. Lüders, M. A. L. Marques, C. Franchini, E. K. U. Gross, A. Continenza, and S. Massidda, Phys. Rev. Lett. 94, 037004 (2005).

[18] R. Arita, T. Koretsune, S. Sakai, R. Akashi, Y. Nomura, and W. Sano, Adv. Mater. 29, 1602421 (2017).

[19] A. Sanna, G. Profeta, A. Floris, A. Marini, E. K. U. Gross, and S. Massidda, Phys. Rev. B 75, 020511(R) (2007).

[20] R. S. Gonnelli, D. Daghero, D. Delaude, M. Tortello, G. A. Ummarino, V. A. Stepanov, J. S. Kim, R. K. Kremer, A. Sanna, G. Profeta, and S. Massidda, Phys. Rev. Lett. 100, 207004 (2008).

[21] A. Floris, A. Sanna, S. Massidda, and E. K. U. Gross, Phys. Rev. B 75, 054508 (2007).

[22] A. Sanna, C. Franchini, A. Floris, G. Profeta, N. N. Lathiotakis, M. Lüders, M. A. L. Marques, E. K. U. Gross, A. Continenza, and S. Massidda, Phys. Rev. B 73, 144512 (2006).

[23] A. Linscheid, A. Sanna, A. Floris, and E. K. U. Gross, Phys. Rev. Lett. 115, 097002 (2015).

[24] A. Floris, A. Sanna, M. Lüders, G. Profeta, N. Lathiotakis, M. Marques, C. Franchini, E. Gross, A. Continenza, and S. Massidda, Physica (Amsterdam) 456C, 45 (2007).

[25] M. Kawamura, R. Akashi, and S. Tsuneyuki, Phys. Rev. B 95, 054506 (2017).

[26] M. Marques, Density functional theory for superconductors, exchange and correlation potentials for inhomogeneous systems, Ph.D. thesis, Bayerische Julius-Maximilians Universität Würzburg, 1998.

[27] L. J. Sham and M. Schlüter, Phys. Rev. Lett. 51, 1888 (1983).

[28] S. Massidda, F. Bernardini, C. Bersier, A. Continenza, P. Cudazzo, A. Floris, H. Glawe, M. Monni, S. Pittalis, G. Profeta, A. Sanna, S. Sharma, and E. K. U. Gross, Supercond. Sci. Technol. 22, 034006 (2009).

[29] S. Baroni, S. de Gironcoli, A. Dal Corso, and P. Giannozzi, Rev. Mod. Phys. 73, 515 (2001). 
[30] See Supplemental Material at http://link.aps.org/ supplemental/10.1103/PhysRevLett.125.057001 for accuracy tests, which includes Ref. [31-33].

[31] P. Giannozzi et al., J. Phys. Condens. Matter 21, 395502 (2009).

[32] I. Errea, M. Calandra, C. J. Pickard, J. Nelson, R. J. Needs, Y. Li, H. Liu, Y. Zhang, Y. Ma, and F. Mauri, Phys. Rev. Lett. 114, 157004 (2015).

[33] Y. Wang and A. Chubukov, Phys. Rev. B 88, 024516 (2013).

[34] F. Marsiglio, Phys. Rev. B 98, 024523 (2018).

[35] A. Sanna, S. Pittalis, J. K. Dewhurst, M. Monni, S. Sharma, G. Ummarino, S. Massidda, and E. K. U. Gross, Phys. Rev. B 85, 184514 (2012).

[36] For the electronic part of both functionals we use the same approximation, $\mathcal{K}^{e e}$.

[37] J. Schmidt, C. L. Benavides-Riveros, and M. A. L. Marques, Phys. Rev. B 99, 224502 (2019).

[38] N. Bergeal, V. Dubost, Y. Noat, W. Sacks, D. Roditchev, N. Emery, C. Hérold, J.-F. Marêché, P. Lagrange, and G. Loupias, Phys. Rev. Lett. 97, 077003 (2006).

[39] C. Kurter, L. Ozyuzer, D. Mazur, J. F. Zasadzinski, D. Rosenmann, H. Claus, D. G. Hinks, and K. E. Gray, Phys. Rev. B 76, 220502(R) (2007).

[40] N. Hauptmann, M. Becker, J. Kröger, and R. Berndt, Phys. Rev. B 79, 144522 (2009).

[41] M. R. Eskildsen, M. Kugler, S. Tanaka, J. Jun, S. M. Kazakov, J. Karpinski, and O. Fischer, Phys. Rev. Lett. 89, 187003 (2002).

[42] J. Geerk, G. Linker, and R. Smithey, Phys. Rev. Lett. 57, 3284 (1986).

[43] A. Cassinese, M. Iavarone, R. Vaglio, M. Grimsditch, and S. Uran, Phys. Rev. B 62, 13915 (2000).

[44] N. Hauptmann, M. Becker, J. Kröger, and R. Berndt, Phys. Rev. B 79, 144522 (2009).

[45] J. Schumann, Phys. Status Solidi B 99, 79 (1980).

[46] T. Takasaki, T. Ekino, and T. Muranaka, Physica (Amsterdam) 378-381C, 229 (2002).

[47] P. Townsend and J. Sutton, Phys. Rev. 128, 591 (1962).

[48] G. Rochlin, Phys. Rev. 153, 513 (1967).

[49] Y. Bugoslavsky, Y. Miyoshi, G. K. Perkins, A. V. Berenov, Z. Lockman, J. LMacManus-Driscoll, L. F. Cohen, A. D. Caplin, H. Y. Zhai, M. P. Paranthaman, H. M. Christen, and M Blamire, Supercond. Sci. Technol. 15, 526 (2002).

[50] T. W. Heitmann, S. D. Bu, D. M. Kim, J. H. Choi, J. Giencke, C. B. Eom, K. A. Regan, N. Rogado, M. A. Hayward, T. He, J. S. Slusky, P. Khalifah, M. Haas, R. J. Cava,
D. C. Larbalestier, and M. S. Rzchowski, Supercond. Sci. Technol. 17, 237 (2004).

[51] B. Blackford, J. Low Temp. Phys. 23, 43 (1976).

[52] R. Reed and A. Boyer, J. Low Temp. Phys. 24, 35 (1976).

[53] P. Richards and M. Tinkham, Phys. Rev. 119, 575 (1960).

[54] D. Finnemore and D. Mapother, Phys. Rev. 140, A507 (1965).

[55] R. Gasparovic, B. Taylor, and R. Eck, Solid State Commun. 4, 59 (1966).

[56] B. Schöneich, D. Elefant, P. Otschik, and J. Schumann, Phys. Status Solidi 91, 99 (1979).

[57] B. Blackford, Physica (Amsterdam) 55, 475 (1971).

[58] B. Blackford and R. March, Phys. Rev. 186, 397 (1969).

[59] I. Giaever, Phys. Rev. Lett. 5, 147 (1960).

[60] R. Hackl, R. Kaiser, and S. Schicktanz, J. Phys. C 16, 1729 (1983).

[61] R. S. Gonnelli, D. Daghero, D. Delaude, M. Tortello, G. A. Ummarino, V. A. Stepanov, J. S. Kim, R. K. Kremer, A. Sanna, G. Profeta, and S. Massidda, Phys. Rev. Lett. 100, 207004 (2008).

[62] J. P. Perdew, K. Burke, and M. Ernzerhof, Phys. Rev. Lett. 77, 3865 (1996).

[63] A preliminary version of the present functional has been used in Refs. [64-71].

[64] A. Sanna, A. V. Fedorov, N. I. Verbitskiy, J. Fink, C. Krellner, L. Petaccia, A. Chikina, D. Y. Usachov, A. Grüneis, and G. Profeta, 2D Mater. 3, 025031 (2016).

[65] C. Pellegrini, H. Glawe, and A. Sanna, Phys. Rev. Mater. 3, 064804 (2019).

[66] J. A. Flores-Livas, A. Sanna, and E. K. U. Gross, Eur. Phys. J. B 89, 63 (2016).

[67] J. A. Flores-Livas and A. Sanna, Phys. Rev. B 91, 054508 (2015).

[68] J. A. Flores-Livas, M. Amsler, C. Heil, A. Sanna, L. Boeri, G. Profeta, C. Wolverton, S. Goedecker, and E. K. U. Gross, Phys. Rev. B 93, 020508 (2016).

[69] I. Errea, F. Belli, L. Monacelli, A. Sanna, T. Koretsune, T. Tadano, R. Bianco, M. Calandra, R. Arita, F. Mauri, and J. A. Flores-Livas, Nature (London) 578, 66 (2020).

[70] J. A. Flores-Livas, A. Sanna, A. P. Drozdov, L. Boeri, G. Profeta, M. Eremets, and S. Goedecker, Phys. Rev. Mater. 1, 024802 (2017).

[71] M. Monni, F. Bernardini, A. Sanna, G. Profeta, and S. Massidda, Phys. Rev. B 95, 064516 (2017). 